\title{
Algunos datos biológicos sobre la trucha común en León
}

\author{
Josefa Domínguez Carrillo y Francisco J. Purroy Iraizoz
}

Departamento de Zoología. Facultad de Biología Universidad de León

\author{
SUMMARY \\ SOME BIOLOGICAL DATA ABOUT THE BROWN TROUT IN LEON (SPAIN)
}

\begin{abstract}
At the first stage in the work that we are carrying out on feeding of Salmo trutta fario L., we present data obtained from the study of fifty-four stomachs removed from specimens caught in the river network of León (NW Spain) during the months of March, April, May and June of 1979 and February 1981. The research period was characterized by three trout mortalities due to different antropogenic causes. It is outstanding that the only empty stomachs collected were those of the fish affected by the mortality of 1981 .

From the results obtained, we deduced that the trout populations studied were mainly bottom feeders. The importance of the different groups of preys changes depending on whether or not we take into account the number of preys items or the percentage of the total volume of these items.

Some comments on the varying degree of the fishing success over the last eighteen years are also added, including a drastic decline of the daily catch and of the average fish weight.
\end{abstract}

\section{INTRODUCCION}

En esta comunicación queremos dar a conocer un primer avance del trabajo que estamos realizando sobre alimentación de trucha común, (Salmo trutta fario L.). Presentamos los resultados obtenidos del estudio de cincilenta y cuatro estómagos, recogidos:de ejemplares capturados en la red fluvial de León durante los meses de marzo, abril, mayo y junio de 1979 y febrero de 1981 .

Estos datos biológicos se completan con noticias sobre tres mortandades de truchas debidas a diferentes causas, y se añaden comentarios sobre la variación del rendimiento pesquero en los últimos años.

\section{ESTUDIOS DE LA ALIMENTACION}

\section{MATERIAL Y METODOS}

Se han estudiado 54 estómagos de truchas 34 de los cuales eran ejemplares capturados desde marzo a junio de 1979 con cebo natural en los ríos Esla (Quintanilla de Rueda), 
Curueño (Nocedo y La Vecilla), Porma (Villafruela del Condado y Vegamián) y Torrestio-Luna (San Emiliano), otros 20 estómagos fueron extraídos de ejemplares del rio Porma durante febrero de 1981 (Candanedo, Lugán, Villafruela del Condado y Palazuelo de Boñar). Todos estos ejemplares estaban muertos o enfermos.

Para el analisis de los contenidos estomacales hemos empleado un método que utiliza simultáneamente el número, frecuencia y volumen de las diferentes presas ingeridas. Para ello, y basándonos en las revisiones metodológicas de HYNES (1950), SORBE (1972) y HERNANDO CASAL (1978) hemos combinado los métodos numéricos y volumétricos expresando los resultados como presencias totales, frecuencias relativas y el porcentaje en volumen de las presas. Para las frecuencias estableceremos tres grupos de acuerdo con SORBE (1972):

Presas accidentales (presentes en menos de un. $10 \%$ de los casos), presas secundarias (entre un 10 y un $50 \%$ ) y presas preferenciales (más de $50 \%$ de los estómagos).

\section{RESULTADOS}

La tabla“I muestra los diferentes grupos de presas de acuerdo con las clases de frecuencia definidas en la dieta de los ejemplares examinados. Aunque las presas preferenciales son parte indudablemente fundamental en la alimentación de la trucha creemos que los otros dos grupos, presas secundarias y accidentales pueden verse modificados al ampliar el número de muestras.

Todos los ejemplares de Efemerópterios, Plecópteros, Tricópteros y Dípteros que hemos encontrado pertenecen a estados larvarios y son de habitat bentónico. Según estos resultados se deduce que las truchas estudiadas se alimentan fundamentalmente de fauna de fondo, resultados que concuerdan con los obtenidos por FROST \& BROWN (1971) y ALVAREZ PELLITERO (1979). Esta última autora señala que no se observan variaciones en la composición de la ali- mentación en los distintos tramos estudiados.

En el orden de importancia de las presas en la dieta, según su frecuencia de aparición, se observa en la tabla II. Si consideramos el porcentaje volumétrico, (ver tabla II) mayor importancia tendrín los Tricópteros, seguidos de Dípteros, Plecópteros, Efemerópteros, Himenópteros, Coleópteros Megalópteros y Miriápodos, Moluscos, Arácnidos, $\mathrm{He}$ mípteros y Nemátodos.

Por último, y por su presencia numérica, la ordenación sería: Dípteros, Tricópteros, Plecópteros, Efemerópteros, Himenópteros, Coleópteros, Moluscos, Megalópteros, Hemípteros, Arácnidos, Miriápodos y Nemátodos. (tabla II y Apéndice).

\section{DISCUSION}

Las diferencias que encontramos con el trabajo de ALVAREZ PELLITERO (1979) no son muy acusadas, ya que en aquél, Tricópteros, Dipteros, Efemerópteros y Plecópteros eran por este orden los componentes fundamentales de la dieta. Creemos que las diferencias se deben a que la citada autora utiliza para el conocimiento de la dieta todo el contenido gastroentérico, lo que produce como consecuencia una desviación tanto en número como en volumen a favor de las presas de digestibilidad menor. La citada autora pretendía obtener el máximo espectro de presas potenciales, con el fin de disponer de datos para el estudio de los ciclos de los parásitos intestinales de la trucha, más que el conocimiento de las distintas proporciones de presas.

Por lo que se refiere al segundo grupo de estómagos, recogidos en el río Porma en febrero de 1981, se encontraron todos vacios, lo que contrasta con los trabajos consultados, y apuntamos como explicación el proceso patológico que parecieron sufrir estos ejemplares (Según comunicación oral del Departamento de Patología Infecciosa de la Facultad de Veterinaria de la Universidad de León). 
TABLA I. Clasificación de las presas según el método de frecuencia. El número total de presas fue de 4.117.

PRESAS ACCIDENTALES

Gastèrópodos $(8,82 \%)$

Hemípteros $\quad(2,94 \%)$

Megalópteros $(2,94 \%)$

Arácnidos $\quad(2,94 \%)$

Miriápodos $\quad(2,94 \%)$

Nemátodos $\quad(2,94 \%)$
PRESAS SECUNDARIAS

Himenópteros $(17,64 \%)$

Coleópteros $(14,70 \%)$
PRESAS PREFERENCIALES

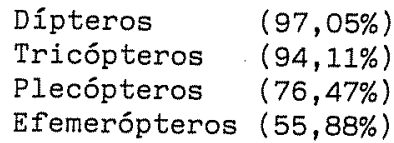

Dípteros $\quad(97,05 \%)$

Plecópteros $\quad(76,47 \%)$

Efemerópteros $(55,88 \%)$

\section{MORTANDADES DE TRUCHAS}

El invierno 1980-81 se ha caracterizado por tres mortandades piscícolas. Una de ellas fue en el río Orbigo, desde Veguellina de Orbigo hasta La Bañeza. La causa fue un vertido de contaminantes derivados del lavado de remolacha en una azucarera de Veguellina. Se valoran oficialmente los peces envenenados en $200 \mathrm{Kgs}$. de truchas y barbos por kilómetro. En otro caso fue en el río Porma, desde el Embalse de Vegamián a Villafruela del Condado. En este caso las truchas muertas presentan infección por Pseudomo-

TABLA II. Resultados obtenidos en el estudio de los contenidos estomacales $(N=34)$.

PRESA

MOLUSCOS

rasterópodos

INEECTOS

Efemerópteros

Plecópteros

Tricópteros

Dipteros

Coleópteros

Hemípteros

Megalópterus

Himenópteros

ARACNIDOS

MIRIAPODOS

NEMATODOS

Materia vegetal inidentificable Materia animal inidentificable Materia mineral inidentificable
PRESENCIAS FRECUENCIA VOLUMETRICO NUMERICO

\begin{tabular}{|c|c|c|c|}
\hline 3 & 8,82 & 0,149 & 0,14 \\
\hline 19 & 55,88 & 4,97 & 2,57 \\
\hline 26 & 76,47 & 16,15 & 3,38 \\
\hline 32 & 94,11 & 32,99 & 14,59 \\
\hline 33 & 97,05 & 18,57 & 78,74 \\
\hline 5 & 14,70 & 0,57 & 0,17 \\
\hline 1 & 2,94 & 0,04 & 0,02 \\
\hline 1 & 2,94 & 0,32 & 0,04 \\
\hline 6 & 17,64 & 0,68 & 0,24 \\
\hline 1 & 2,94 & $0,10 /$ & 0,02 \\
\hline 1 & 2,94 & 0,32 & 0,02 \\
\hline 1 & 2,94 & 0,02 & 0,02 \\
\hline 6 & 17,64 & 1,199 & - \\
\hline 6 & 76,47 & $23,84^{\circ}$ & - \\
\hline 1 & 2,94 & 0,02 & - \\
\hline
\end{tabular}




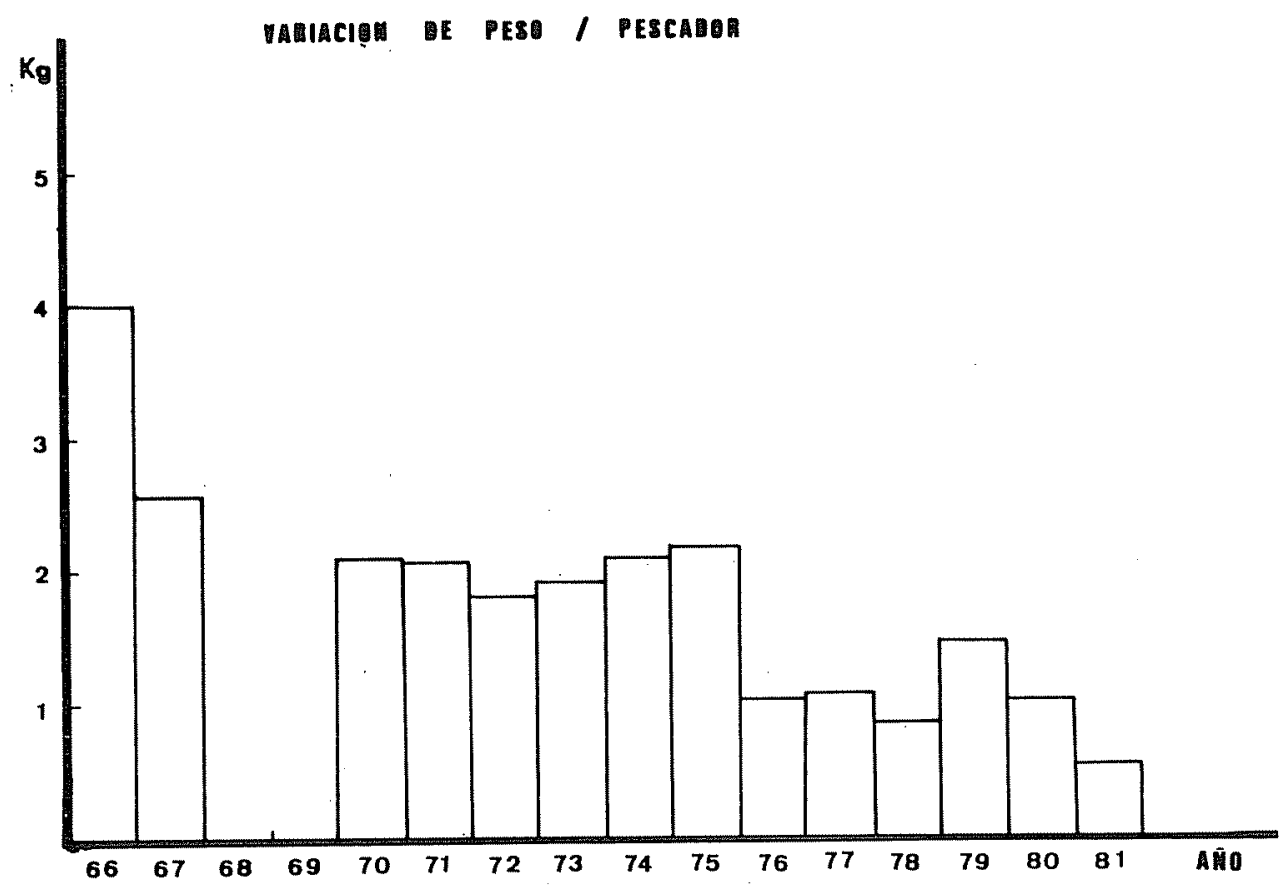

Fig. 1. Variación del peso capturado por pescador a lo largo de diferentes años.

nas y Saprolegnia, según diagnóstico emitido por el Departamento de Patología Infecciosa de la Facultad de Veterinaria de León. La mortandad se inicia en las inmediaciones de una piscifactoría de trucha arco iris de Palazuelo de Boñar propagándose aguas arriba y abajo, lo que coincide con un estiaje muy acusa do del río. Debido precisamente a este estiaje la dilución de detergentes, lejías y vertidos fecales es inferior a la normal por lo que los agentes causantes de la enfermedad se vieron favorecidos.

En este río en las rejillas de los canales de dos centrales (Lugán, Carrizal) donde desde final de octubre de 1980 a febrero de 1981 se recogió una cantidad variable entre 6-10 Kgs. de trucha por dia. En un inventario realizado en Lugán, el 13-II-81, se prospectaron $1,5 \mathrm{Km}$. de cauce observándose 14 individuos muertos, 7 enfermos, sin" reflejo de huida, y sólo dos truchas sanas. Aún con- siderando el valor indicativo de esta prospección visual, la mortandad puede considerarse elevadísima. A partir de la confluenciá con el río Curueño, de caudal más limpio, disminuyó la cifra de ejemplares muertos varados en la orilla.

El tercer caso se produjo en el río Bernesga, de Villamanín a La Robla. La causa de la mortandad es similar a la anterior y se detectó además Aeromonas hidrophylla. No se han cuantificado sus consecuencias. Estas hecatombes comprobadas advierten una degradación inquietante de las aguas en la red hidrográfica leonesa.

\section{VARIACION DEL RENDIMIENTO PESQUERO}

Por último, y sobre la variación del rendi- 


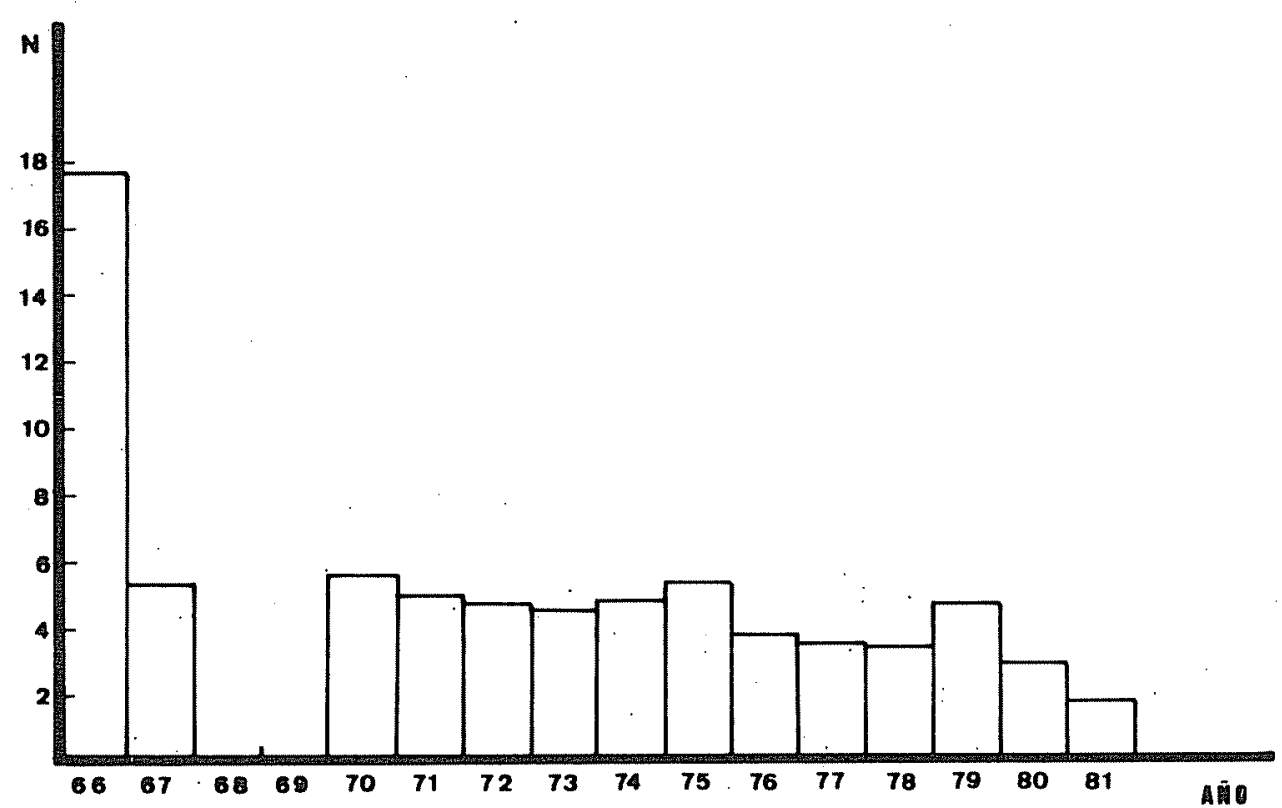

Fig. 2. Número de ejemplares cap turados por pescador a lo largo de difer entes años.

miento pesquero, presentamos las gráficas de variación de peso por pescador y variación del número de ejemplares por pescador (figs. 1 y 2).

Para la realización de estas gráficas hemos trabajado con datos de captura de la "Semana Internacional de la Trucha", que celebró este año su XVI edición. Para conseguir estos datos consultamos las hemerotecas de los periódicos locales y los archivos de la organización del concurso. Este concurso se desarrolla en cuatro días de pesca, correspondientes a tres fases selectivas y una final. Los lugares de pesca son los mejores cotos de la provincia.

De la observación de los histogramas podemos concluir que tanto el número de truchas como el peso por pescador desciende a lo largo del tiempo en que se viene desarro- llando el concurso (figs. 1 y 2). Las diferencias no son muy abruptas si se comparan dos años consecutivos, pero comparando el primero, el octavo y el decimosexto estas diferencias se acentúan. En resumen, el cotejo del número de capturas y peso de las mismas sugiere una evolución interanual en continuo declive, sin ninguna duda relacionada con la disminución del capital íctico.

\section{AGRADECIMIENTOS}

Agradecemos la colaboración del Dr. Cármenes del Dpto de Patología Infecciosa de la Facultad de Veterinaria de León, asi como a los organizad ores de la Semana Internacional de la Trucha. 
ALVAREZ PELLITERO, M.P. 1979. Helmintosis de la trucha en Leon. Institución "Fray Bernardino de Sahaguin" de la Excma. Diputación Provincial. (C.S.I.C.) León.

FR.OST, W.E. \& BROWN, M.E. 1971. La trucha. Ėd. Academia. León.

HERNANDO CASAL, JA. 1978. Estructura de lo Comunidad de Peces de la Marisma del Guadalquivit. Tesis doctoral. Universidad de Sevilla.
HYNES, H.B.N. 1950. The food of fresh-water stiklebacks (Gasterosteus aculeatus y Pygosteus pungitius), with a reviev of methods used in studies of the food of fishes. J. Anim. Ecol., 19: $36: 58$.

SORBE, J.C. 1972. Ecologie et éthologie alimen taire de l'ichthyo-faune chalutable du plateau continental sud Gascogne. Thèse de 3ème. cycle. Univ. Aix-Marseille. 125 pàgs.

\section{APENDICE}

Relación de presas encontradas en los contenidos estomacales.

PRESEN - FRECUEN-

$\begin{array}{cc}\% \\ \text { VOLUMEN cc. } \% & \text { EJEMPLARES }\end{array}$ $\%$

GASTROPODA

Ancylidae

3

8,82

0,07

0,149

6

0,145

COLEOPTERA

Dytiscidae Agabus sp.

Elateridae EZater sp.

Elmidae

EZmis sp.

Staphilinidae

Larva sin determinar

1

2,94

0,13

0,278

1

0,024

$1 \quad 2,94$

0,05

0,107

2,94

0,02

0,042

2,94

0,03

0,064

2,94

0,01

0,021

0,03

0,064

0,024

$1 \quad 2,94$

DIPTERA

Blepharoceridae

$1 \quad 2,94$

0,01

0,021

94,11

8,23

17,634

Dolichopodidae

2,94

0,05

0,107

Simulidae

Tabanidae

44,11

0,35

0,749

0,064

0,048

0,024

0,024

2

5,88

0,03

0,024

EPHEMEROPTERA

Baëtidae

Baëtis sp.

Ecdyonuridae

Ecdyonurus sp.

Rhithrogena sp.

$2 \quad 5,88$

0,04

0,085

0,67

1,435

40

0,024

3203

77,89

$9 \quad 26,47$

0,63

1,349

0,98

2,099

16

0,024

30

0,729

$4 \quad 11,76$

48

0,072

HETEROPTERA

Notonectidae

Notonecta sp.

2,94

0,02

0,042

1

0,024 


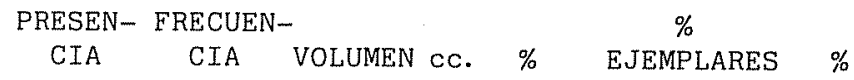

Perlodidae

Isoperta sp.

$\begin{array}{lllllll}9 & 26,47 & 0,89 & 1,907 & 72 & 1,750\end{array}$

TRICHOPTERA

Brachycentridae

Micrasema sp.

Goeridae

Larcasia sp.

Hydropsychidae

Hydropsyche sp.

Limnophilidae '

Linmephilus sp.

Alzogamus sp.

Potamophylax sp.

Anomalopterygella sp.

Psychomyiidae

Psychomyia sp.

Rhyacophilidae

Rhyacophila sp.

Sericostomatidae

ARANEAE

Drassidae

SCOLOPENDROMORPHA

Scolopendridae

Scolopendra sp.

NEMATODA

Sin identificar

HIMENOPTERA

Formicidae

Ichneumonidae

Megachilidae

$\begin{array}{rrllrl}24 & 70,58 & 3,44 & 7,370 & 317 & 7,709 \\ 1 & 2,94 & 0,05 & 0,107 & 1 & 0,024 \\ 7 & 20,58 & 0,92 & 1,971 & 18 & 0,437 \\ 5 & 14,70 & 2,0 & 4,285 & 21 & 0,510 \\ 1 & 2,94 & 0,03 & 0,064 & 2 & 0,048 \\ 1 & 2,94 & 1,8 & 3,856 & 10 & 0,243 \\ 2 & 5,88 & 1,0 & 2,142 & 2 & 0,048 \\ 20 & 58,82 & 3,2 & 6,856 & 189 & 4,596 \\ & & & & & 0,145 \\ 3 & 8,82 & 0,07 & 0,149 & 6 & 0,14 \\ & & & & & \\ 15 & 44,11 & 1,08 & 2,314 & 22 & 0,535 \\ 4 & 11,76 & 1,36 & 2,914 & 12 & 0,291\end{array}$

$\begin{array}{llllll}1 & 2,94 & 0,05 & 0,107 & 1 & 0,024\end{array}$

MEGALOPTERA

Sialidae

sialis sp.

$1 \quad 2,94$

0,15

0,321

0,048

PLECOPTERA

Capniidae

Capnia sp.

Chloroperlidae

Siphonoperla sp.

Leuctridae

Leuctra sp.

Nemouridae

Amphinemura sp.

Protonemura sp.

Perlidae

Dinocras sp.

$\begin{array}{rrllll}1 & 2,94 & 0,01 & 0,021 & 1 & 0,024 \\ 2 & 5,88 & 0,1 & 0,214 & 2 & 0,048 \\ 4 & 11,76 & 0,18 & 0,385 & 14 & 0,340 \\ 2 & 5,88 & 0,16 & 0,342 & 16 & 0,389 \\ 5 & 14,70 & 0,09 & 0,192 & 8 & 0,194 \\ 2 & 5,88 & 0,04 & 0,085 & 2 & 0,048 \\ 13 & 38,23 & 6,07 & 13,006 & 29 & 0,705\end{array}$

